\title{
COMPORTEMENT DU CUIVRE ET DE SES ALLIAGES
}

\author{
P. ANCRENAZ ${ }^{*}$ et C. SERVANT* * \\ * DGA/CREA, 16 bis avenue prieur de la côte d'Or, F-94114 \\ Arcueil Cedex, France \\ * ISMA, Laboratoire de Métallurgie Structurale, Unité \\ Associée au CNRS $N^{\circ} 1107$, Université Paris-Sud, Bátiment 413, \\ F-91405 orsay Cedex, France
}

\begin{abstract}
Influence of additional elements ( $\mathrm{Si}$ and $\mathrm{Co}$ ) is studied on behavior of copper polycristal. In this purpose, two alloys have been chosen : $\mathrm{Cu} 3 \%$ Si alloy is a solid solution in which silicon atoms are in substitution sites, $\mathrm{Cu} 2 \%$ Co alloy in which cobalt form coherent and spherical precipitates. These materials are tested under static and dynamic compression. Effect of schock on mechanical properties are studied. Deformation mechanisms and relation between deformation microstructure and mechanical behavior are investigated by transmission electronic microscopy.
\end{abstract}

\section{I) INTRODUCTION}

Le cuivre pur a fait l'objet de nombreux travaux tant au niveau de ses propriétés mécaniques (/1/) que microstructurales $(/ 2 /)$. Afin d'évaluer l'influence des éléments d'addition sur le comportement du cuivre en compression, deux alliages modèles ont été élaborés : l'alliage $\mathrm{Cu} 3 \%$ Si dont l'élément est en solution solide dans le cuivre, et l'alliage $\mathrm{Cu} 2 \%$ Co dans lequel le cobalt forme des précipités. Le comportement en plasticité de ces matériaux est étudié en compression statique et dynamique afin de quantifier la sensibilité à la vitesse de déformation. Par ailleurs, l'effet de l'écrouissage créé par le passage d'une onde de choc est étudié sur les propriétés mécaniques en compression statique et dynamique de ces alliages. Afin de comprendre les différences de comportement de ces matériaux, l'analyse de la microstructure est nécessaire pour analyser les mécanismes de déformation mis en jeu au cours de la déformation.

\section{II) MATERLAUX ET METHODES EXPERIMENTALES}

Les deux alliages étudiés, $\mathrm{Cu} 2 \% \mathrm{Co}$ et $\mathrm{Cu} 3 \% \mathrm{Si}$, ont été élaborés à partir de cuivre de pureté $5 \mathrm{~N}$. Après le traitement thermique de recristallisation, la taille de grains du cuivre pur et de l'alliage $\mathrm{Cu} 3 \%$ Si est de 100 microns alors qu'elle est de 500 microns environ pour l'alliage $\mathrm{Cu} 2 \% \mathrm{Co}$. En effet, dans le cas de cet alliage, un recuit d'homogénéisation $\left(1 \mathrm{~h} . \grave{a} 960^{\circ} \mathrm{c}\right)$ qui entraine un fort grossissement des grains, a précédé le revenu de précipitation $\left(3 \mathrm{~h}\right.$. à $650^{\circ} \mathrm{c}$ ). Ce dernier détermine la taille des précipités de cobalt et la nature de l'interface précipité matrice (cohérente ou incohérente).

Trois types d'essais mécaniques ont été effectués;

- compression statique $\left(\dot{\varepsilon}=5.10^{-4} \mathrm{sec}^{-1}\right)$

- compression dynamique ( $\dot{\varepsilon}$ variant de 1000 à $4000 \mathrm{sec}^{-1}$ ) : les essais ont été réalisés grâce à un système de barre d'Hopkinson qui permet d'établir les courbes contraintes déformation et de fixer le taux de déformation de l'éprouvette au moyen de bagues d'arrèt, pour les anlyses microstructurales.

- choc par impact de plaque : les matériaux ont été testés à deux pressions de choc, 10 et $20 \mathrm{GPa}$. L'échantillon a été placé dans un dispositif dont la géométrie limite les phénomènes de détente latérale et d'écaillage en face arrière. 
III) INFLUENCE DE LA VITESSE DE SOLLICITATION SUR LES PROPRIETES DU CUIVRE ET DES DEUX ALLIAGES EN COMPRESSIQN

\section{III-1) ETUDE DU CUIVRE PUR}

\section{a) Essais mécaniques}

En sollicitation statique, la courbe contrainte déformation du cuivre pur (fig. $n^{\circ} 1$ ) indique une forte consolidation, attribuée au mécanisme de déformation qui est un mécanisme d'intersection de dislocations thermiquement activé. En mode dynamique (fig. $n^{\circ}$ ), l'augmentation de la vitesse de déformation $(\dot{\varepsilon}=1000$ $\mathrm{sec}^{-1}$ ) se traduit par l'augmentation du niveau des contraintes d'écoulement et du taux de consolidation. Ainsi l'écart entre les contraintes d'écoulement en mode statique et dynamique augmente à mesure que la déformation croît.

La mesure de l'énergie emmagasinnée par déformation plastique grace à l'analyse calorimétrique différentielle, donne un élément de réponse. En effet, la valeur de cette énergie est plus importante en mode dynamique qu'en mode statique (fig. $n^{\circ} 3$ ). Pour mettre en évidence cette propriété, il convient de limiter l'échauffement qui se produit au sein de l'éprouvette au cours de la déformation en mode dynamique, et dans ce but, l'échantillon est déformé par étapes successives de $25 \%$ chacune. Ainsi, l'échantillon emmagasine de l'énergie de manière linéaire avec la déformation plastique en régime dynamique. Mais lorsque l'échantillon est déformé en une seule fois, grace à l'échauffement subi par le matériau en mode dynamique, il y a apparition d'un phénomène de restauration dynamique, et cela se traduit par l'apparition d'un plateau sur la courbe $E=f\left(\varepsilon_{p}\right)$, pour un taux de déformation plastique vraie de l'ordre de 0,4 .

Fig. $n^{\circ} 3$ :

variation de l'energie emmagasinnée en fonction du taux de deformation

o compression dynamique par étapes

compression dynamique

$\Delta$ compression statique

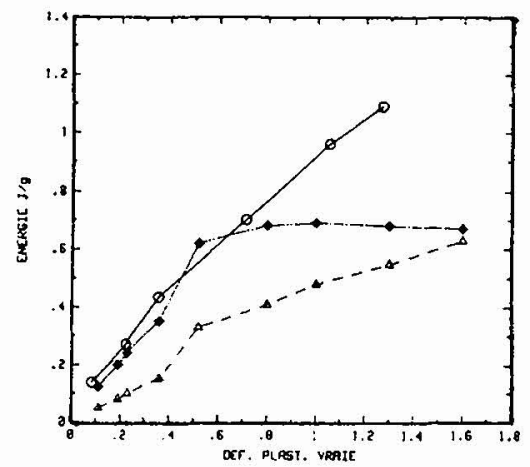

b) microstructure de déformation

En compression statique, les microstructures de déformation du cuivre révélées par microscopie électronique en transmission (MET) montrent que les dislocations s'arrangent en cellules de dislocations(Photo $\left.n^{\circ} 1\right)$. La taille de ces cellules diminue lorsque le taux de déformation augmente. En compression dynamique, les différences d'arrangement de ces dislocations sont faibles, cependant la structure des cellules de dislocations n'est pas aussi bien définie que sous sollicitation statique car, notamment, les parois de ces cellules sont plus larges et parfois incomplètes.

Photo $n^{\circ} 1$ :

cellules de dislocations présentées par le cuivre pour un taux de déformation de $20 \%$ en compression dynamique

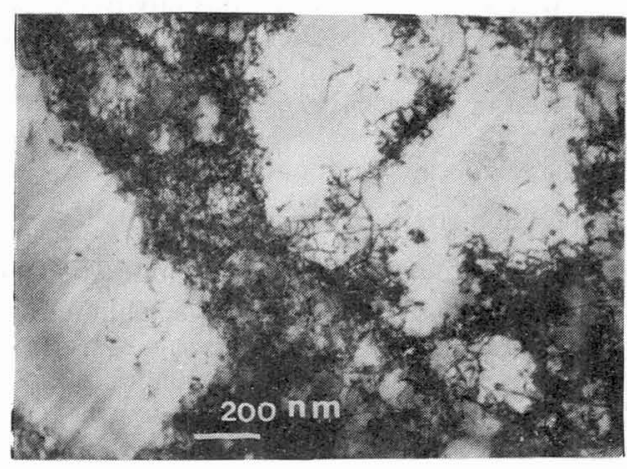




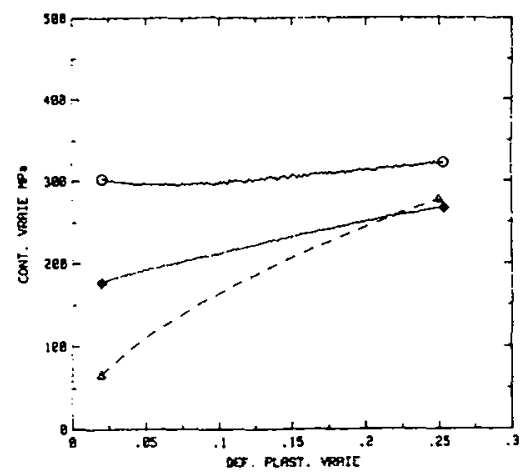

Fig. $n^{\circ} 1$ :

Compression statique du cuivre pur

o cuivre choqué à $20 \mathrm{GPa}$

- cuivre choqué à $10 \mathrm{GPa}$

$\Delta$ cuivre non choqué

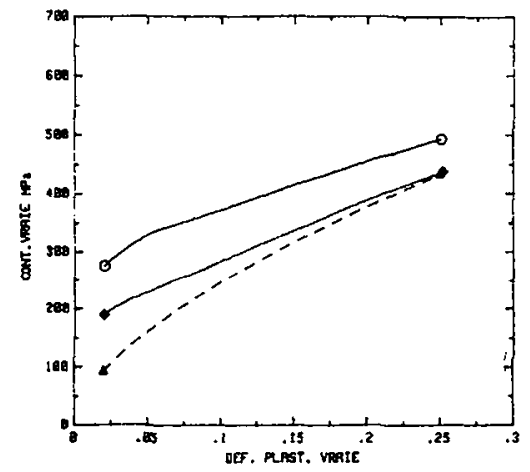

Fig. $n^{\circ} 4$ :

Compression statique de l'alliage $\mathrm{Cu} 3 \% \mathrm{Si}$

- $\mathrm{Cu} 3 \%$ Si choqué à $20 \mathrm{GPa}$

- Cu $3 \%$ Si choqué à $10 \mathrm{GPa}$

$\Delta \mathrm{Cu} 3 \%$ Si non choqué

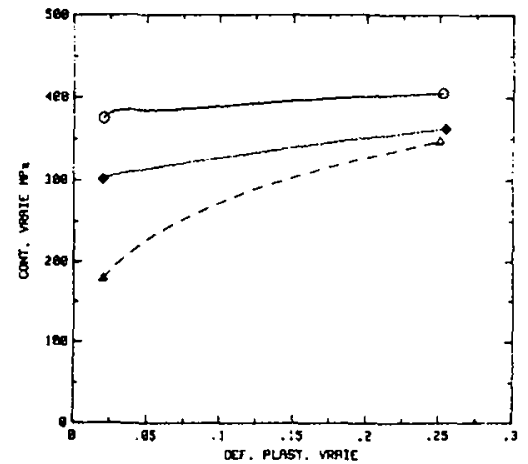

Fig. $n^{\circ} 6$ :

Compression statique de l'alliage $\mathrm{Cu} 2 \% \mathrm{Co}$

- Cu $2 \%$ Co choqué à $20 \mathrm{GPa}$

- $\mathrm{Cu} 2 \%$ Co choqué à $10 \mathrm{GPa}$

$\Delta \mathrm{Cu} 2 \%$ Co non choqué

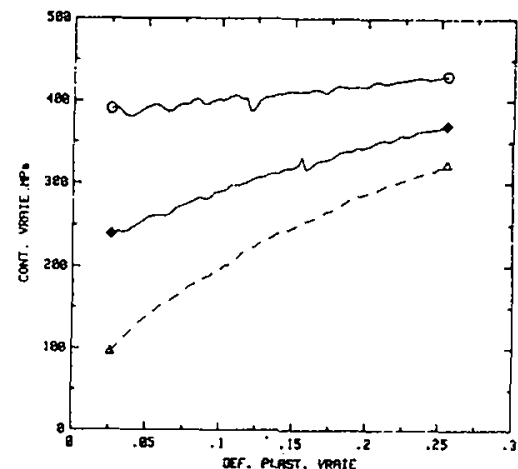

Fig. $n^{\circ} 2$ :

Compression dynamique du cuivre pur

o cuivre choqué à $20 \mathrm{GPa}$

- cuivre choqué à $10 \mathrm{GPa}$

$\Delta$ cuivre non choqué

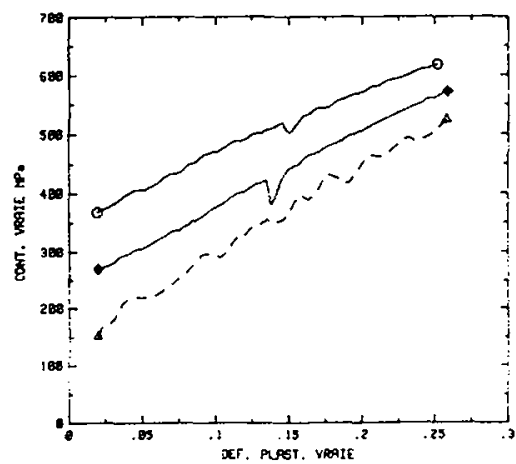

Fig. $n^{\circ} 5$ :

Compression dynamique de l'alliage $\mathrm{Cu} 3 \% \mathrm{Si}$

- $\mathrm{Cu} 3 \%$ Si choqué à $20 \mathrm{GPa}$

- Cu 3\% Si choqué à $10 \mathrm{GPa}$

$\triangle \mathrm{Cu} 3 \% \mathrm{Si}$ non choqué

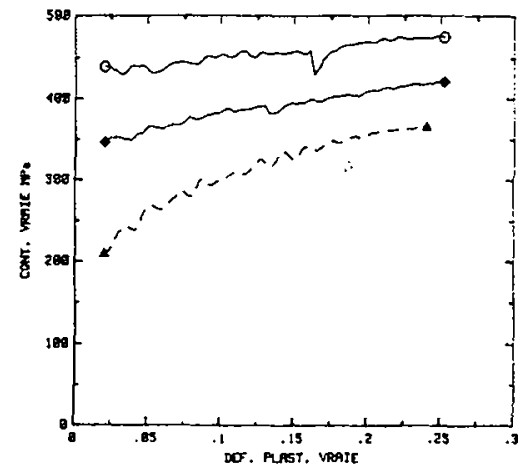

Fig. $n^{\circ} 7$ :

Compression dynamique de l'alliage $\mathrm{Cu} 2 \% \mathrm{Co}$

- $\mathrm{Cu} 2 \%$ Co choqué à $20 \mathrm{GPa}$

- Cu 2\% Co choqué à $10 \mathrm{GPa}$

$\Delta \mathrm{Cu} 2 \%$ Co non choqué 


\section{III-2) EFEET DE L'ENERGIE DE FAUTE D'EMPILEMENT : ALLIAGE CU 3\% SI}

\section{a) essais mécaniques}

L'addition de silicium en très faible quantité diminue trés fortement l'énergie de faute d'empilement (EFE). En effet, l'EFE du cuivre pur est de l'ordre de 50 à $70 \mathrm{~mJ} . \mathrm{m}-2$, alors qu'elle n'est plus que de $10 \mathrm{~mJ} . \mathrm{m}-2$ dans le cas de l'alliage $\mathrm{Cu} 3 \% \mathrm{Si}(/ 3 /)$.

En compression statique, la diminution de l'EFE se traduit par une augmentation de la limite d'élasticité et par une augmentation de la consolidation.(fig. $n^{\circ} 4$ ) Comparé au mode statique, l'effet de la compression dynamique (fig. $n^{\circ}$ ) se traduit par une augmentation de la limite d'élasticité et du niveau des contraintes d'écoulement. La sensibilité à la vitesse de déformation est similaire à celle du cuivre.

b) microstructure de déformation

L'alliage $\mathrm{Cu} 3 \%$ Si présente des microstructures très différentes de celles du cuivre pur (Photo n ${ }^{\circ}$ ), en effet, cet alliage contient des fautes d'empilement, des micromacles, des dislocations parfaites et des dislocations partielles libres. La faible EFE de cet alliage favorise la dissociation des dislocations parfaites en dislocations partielles. La proportion de chacun de ces défauts cristallins dépend de l'orientation cristalline des grains par rapport à l'axe de compression. La déformation s'effectue grace à la propagation des dislocations parfaites et des dislocations partielles car ces dernières créent des fautes d'empilement et lorsque les dislocations partielles se propagent sur des plans superposés, elles créent alors des micromacles $(/ 4 /)$. Il ya donc compétition entre glissement et maclage par dislocations partielles. A. Coujou $(/ 5 /)$ a reporté que les macles peuvent se propager à travers un champ de dislocations et que dès lors, ces macles bloquent le glissement des dislocations parfaites. Ainsi, l'apparition d'une macle au sein d'une zone de glissement inhibe le glissement et favorise le maclage. En effet, les observation en MET révèlent que la proportion de zones déformées par maclage augmentait avec le taux de déformation.

Comparé au cuivre pur, le durcissement de l'alliage $\mathrm{Cu} 3 \% \mathrm{Si}$ semble donc lié à l'apparition du maclage et à la diversification du type d'interaction entre défauts cristallins; interactions dislocations-dislocations, dislocations-macles, macles-macles.

Photo $\mathrm{n}^{\circ} 2$ :

intersections de micromacles présentées par l'alliage $\mathrm{Cu} 3 \% \mathrm{Si}$ pour un taux de déformation de $20 \%$ en compression dynamique

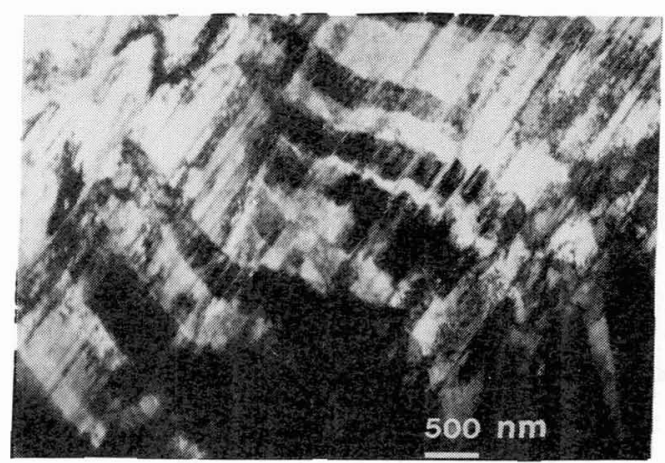

\section{III-3) EFFET DE LA PRECIPITATION : ALLIAGE CU 2\% CO}

\section{a) essais mécaniques}

Le cobalt étant insoluble dans le cuivre à température ambiante, cet alliage subit un revenu de précipitation $\left(3 \mathrm{~h}\right.$. à $650^{\circ} \mathrm{c}$ ) au terme duquel apparaissent des précipités de cobalt sphériques et cohérents avec le réseau cristallin de la matrice de cuivre.

En compression statique (fig. $n^{\circ} 6$ ), l'effet de la précipitation dans le cuivre se traduit par une augmentation très importante de la limite d'élasticité et par une diminution du taux de consolidation. Cependant, l'effet de $l^{\prime}$ augmentation de la vitesse de déformation en compression dynamique (fig. $\mathrm{n}^{\circ} 7$ ) induit une augmentation des valeurs des contraintes d'écoulement qui est relativement faible comparée au cas du cuivre pur. La sensibilité à la vitesse de déformation de cet alliage est plus faible que celle du cuivre pur

b) microstructure de déformation

Pour un taux de déformation de 5\%, les observations effectuées par MET mettent en évidence que certains précipités ne sont plus cohérents avec le réseau cristallin de la matrice (Photo ${ }^{\circ} 3$ ). Ce phénomène s'accentue lorsque le taux de déformation augmente. Il semble donc que les précipités soient cisaillés par les dislocations et deviennent incohérents. La répartition des dislocations est uniforme dans les grains contrairement au cuivre qui présentent des cellules de dislocations

Les précipités sont des obstacles au mouvement des dislocations puisque ces précipités deviennent incohérents et de plus, ils créent une distorsion du réseau cristallin, donc un champ de contrainte autour des 
précipités qui entrave le mouvement des dislocations : ceci conduit à une forte augmentation de la limite d'élasticité.

photo $n^{\circ} 3$ :

microstructure de l'alliage $\mathrm{Cu} 2 \% \mathrm{Co}$

déformé de $10 \%$ en compression dynamique:

certains précipités sont devenus incohérents.

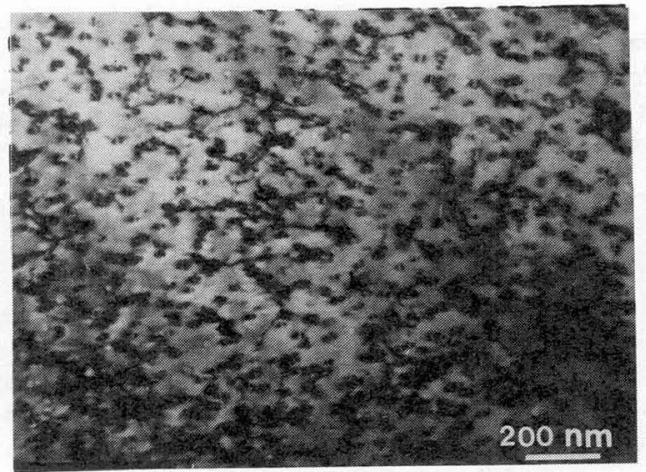

IV) EFFET DU CHOC SUR LE COMPORTEMENT EN COMPRESSION STATIQUE ET DYNAMIQUE DU CUIVRE ET DES DEUX ALLIAGES

L'effet d'un choc sur la microstructure et sur les propriétés mécaniques peut être très important, même si le taux de déformation plastique est très faible. En effet, le passage d'une onde de choc dans un matériau engendre la création d'un grand nombre de défauts cristallins qui n'ont pas toujours le temps de s'arranger dans la configuration la plus stable à cause de la très faible durée du pulse de l'onde de choc.

Le cuivre pur et les deux alliages $\mathrm{Cu} 3 \% \mathrm{Si}$ et $\mathrm{Cu} 2 \% \mathrm{Co}$ ont été testés à deux pressions de choc, 10 et 20 $\mathrm{GPa}$, par une technique d'impact de plaque. Le taux de déformation plastique des échantillons choqués à 10 GPa est de $0,7 \%$, et de $1,4 \%$ pour les échantillons choqués à $20 \mathrm{GPa}$. Les essais en compression statique et dynamique ont permis d'évaluer l'effet de cet ecrouissage sur le comportement en plasticité des matériaux choqués, comparativement aux matériaux non choqués.

\section{IV-1) ETUDE DU CUIVRE PUR}

Les essais en compression statique effectués sur le cuivre pur choqué (fig. $\mathrm{n}^{\circ} 1$ ) présentent une très forte augmentation de la limite d'élasticité. En effet, la contrainte à 0,02 de déformaton plastique vraie, est de 175 MPa pour l'échantillon choqué à $10 \mathrm{GPa}$, et de $300 \mathrm{MPa}$ après un choc de $20 \mathrm{Gpa}$. Par contre, les matériaux choqués présentent une faible consolidation comparée à celle du matériau recristallisé. De plus, le cuivre ayant subi un choc de $20 \mathrm{GPa}$ laisse apparaitre un léger adoucissement qui se traduit par une diminution de $10 \mathrm{MPa}$ de la contrainte pour une déformation plastique vraie de 0,05 . Ce phénomène singulier est peut-être du à la création d'un arrangement instable des dislocations engendré par le passage de l'onde de choc. Cet arrangement de dislocations se restaure sous l'effet de la contrainte externe appliquée et conduit à l'adoucissement observé en compression statique. Cette hypothèse reste à confirmer par analyse calorimétrique différentielle et par analyse par MET.

Le cuivre choqué et testé en compresion dynamique (fig. $\mathrm{n}^{\circ} 2$ ) présente une augmentation de $50 \mathrm{MPa}$ de la limite d'élasticité comparé à la compression statique. Dans le cas du choc à $20 \mathrm{GPa}$, l'adoucissement observé sous sollicitation statique n'apparait pas. Le cuivre choqué présente un taux de consolidation plus important en compression dynamique que statique.

Finalement, l'effet de l'augmentation de la vitesse de déformation semble identique pour le cuivre recristallisé et le cuivre choqué, mais l'écrouissage par choc augmente considérablement le niveau des contraintes d'écoulement et diminue le taux de consolidation.

\section{IV-2)_ALLIAGE CU 3\% SI}

Après choc, l'alliage $\mathrm{Cu} \mathrm{3 \%} \mathrm{Si} \mathrm{conserve} \mathrm{un} \mathrm{taux} \mathrm{de} \mathrm{consolidation} \mathrm{important} \mathrm{en} \mathrm{compression} \mathrm{statique} \mathrm{et}$ dynamique (fig. $n^{\circ} 4, n^{\circ} 5$ ), contrairement au cuivre pur. Cette consolidation semble pouvoir s'expliquer en terme de compétition entre glissement et maclage car la faible EFE de cet alliage implique la mise en jeu de mécanismes de déformation différents de ceux du cuivre pur. Aprés choc, l'état d'écrouissage résiduel est donc stable et conduit à un durcissement important aussi bien en compression statique que dynamique. Enfin, cet alliage présente la même sensibilité au choc que le cuivre pur. 


\section{IV-3) ALLIAGE CU $2 \%$ CO}

En compression dynamique, le durcissement par précipitation induit une forte augmentation de la limite d'élasticité. L'état d'écrouissage crée par le passage de l'onde de choc implique une plus forte augmentation de la limite d'élasticité et une diminution du taux de consolidation (fig. $n^{\circ} 6, n^{\circ} 7$ ). En compression, les matériaux choqués présentent une forme de courbe trés similaire à celle présentée par le cuivre pur, mais à taux d'écrouissage par choc équivalent, les contraintes d'écoulement de l'alliage $\mathrm{Cu} 2 \%$ Co sont plus élevées que celles du cuivre pur.

\section{V) CONCLUSIONS}

En compression dynamique, le niveau des contraintes d'écoulement est plus élevé qu'en compression statique dans le cas du cuivre pur et des deux alliages étudiés. Cette augmentation du niveau des contraintes d'écoulement est liée la densité de dislocations plus importante en compression dynamique que statique. L'état d'écrouissage créé par le passage d'une onde de choc modifie considérablement les propriétés mécaniques et se traduit par une augmentation de la limite d'élasticité d'autant plus importante que la pression de choc est grande. Les matériaux écrouis par choc présentent les mêmes différences de comportement entre compression statique et dynamique, que les matériaux non choqués.

Le cuivre pur se déforme par glissement de dislocations parfaites qui s'arrangent en cellules de dislocations. Les différences de microstructure des échantillons déformés entre compression statique et dynamique sont peu marquées. L'écrouissage créé par un choc de $20 \mathrm{GPa}$ conduirait à la formation d'un arrangement de dislocations instable qui se traduirait par le léger adoucissement observé en compression statique, l'analyse microstructurale par MET restant à effectuer pour valider ou non cette hypothèse.

Les microstructures de l'alliage $\mathrm{Cu} 3 \%$ Si présentent à la fois des micromacles et des dislocations. Il y a compétition entre ces deux mécanismes de déformations, et la participation du maclage à la déformation prend de plus en plus d'importance à mesure que le taux de déformation croît. Ces deux types de mécanisme de déformation sont à l'origine du taux de consolidation très important présenté par cet alliage. Ce matériau choqué, contrairement au cuivre pur conserve ses propriétés de durcissement par écrouissage. Le durcissement par précipitation de l'alliage $\mathrm{Cu} 2 \%$ Co conduit à une augmentation de la limite d'élasticité mais à une diminution du taux de consolidation. Cet alliage accomode la déformation par glissement de dislocations parfaites. L'effet du choc sur les propriétés mécaniques en compression de cet alliage, est similaire à celui du cuivre choqué et se traduit par l'augmentation de la limite d'élasticité et par la diminution du taux de consolidation, cependant, le niveau des contraintes d'écoulement de l'alliage Cu $2 \%$ Co reste plus élevé que dans le cas du cuivre.

\section{REMERCIEMENTS}

Que Mr Gazeaud soit remercier pour la connaissance et l'aide apportées à la réalisation des essais de choc par relèvement de plaques à l'ISL.

\section{REFERENCES}

1// DUJARDiN S. , GAZEAUD G. , LiCHTENBERGER A. ,Journal de Physiques, coll. C3, sup. $n^{\circ} 9$, 49(1988)55

12/ CHEVAL F. , PRIESTER L. Journal de Physiques, coll. C3, sup. n9, 49(1988)47

B/ NORDSTROM T. V., Acta Met. , 17(1969)139

14/ MAHAJAN S. , Acta Met. , 21(1973)1353

15/ COUJOU A. , Phil. Mag. , $56 \mathrm{n}^{\circ} 1(1987) 13$ 